\title{
A NOTE ON THE DARBOUX THEORY OF INTEGRABILITY OF NON-AUTONOMOUS POLYNOMIAL DIFFERENTIAL SYSTEMS
}

\author{
DAVID BLÁZQUEZ-SANZ ${ }^{1}$ AND CHARA PANTAZI ${ }^{2}$
}

\begin{abstract}
In this work we unfold some differential algebraic aspects of Darboux first integrals of polynomial vector fields. An interesting improvement is that our approach can be applied both to autonomous and non-autonomous vector fields. We give a sufficient and necessary condition for the existence of a Darboux first integral of a specific form for a polynomial vector field with some known algebraic invariant hypersurfaces. For the autonomous case, the classical result of Darboux is obtained as a corollary. For the non-autonomous case our characterization improves a known criterium.
\end{abstract}

\section{INTRODUCTION}

One of the most important subjects in mathematical physics is the discussion of integrability of differential equations started by Newton in the 17th century. There is not an unified approach to integrability. There are many different technics for integrating differential equations or proving their non-integrability within some specific framework: Lie symmetries [26], Noether symmetries [4], Lax pairs [14], Painlevé analysis [2], differential Galois theory [24, 28], Darboux's method [10], among others.

A useful approach is the search of invariants and their classification. The first class of invariants are the conserved quantities or first integrals. There are also some other invariants like Jacobi multipliers, tensor invariants or symmetries. The search and classification of those invariant objects of differential equations is still a very active research topic.

In this paper we give some generalization of a classical method started by Darboux in 1878 for the computation of first integrals of polynomial differential systems, $[10,11]$. More recently this method has been refined by several authors, Jouanolou, Singer, Schlomiuk, Llibre, Christopher, Zhang [5, 9, 18, 22, 20, 21, 13, 25, 28, 19, 17, $15]$ among others. This method has been related with some applications concerning limit cycles, centers and bifurcation problems, see for instance [12, 23, 27]. Moreover, several inverse problems for dimension two have been studied in $[7,16,8]$.

Here we isolate the differential algebraic nature of the classical Darboux's method on elementary first integrals. More concretely, in Theorems 5 and 8 we present some new results related to the known theorems about Darboux integrability for autonomous and non-autonomous polynomial vector fields in $\mathbb{C}^{n}$.

2000 Mathematics Subject Classification. Primary 34C14, Secondary 12H05.

Key words and phrases. Darboux first integrals, Jacobi multipliers, non-autonomous polynomial vector fields.

Both authors are supported by the MICIIN/FEDER grant number MTM2009-06973. CP is additionally partially supported by the MICIIN/FEDER grant MTM2008-03437 and by the Generalitat de Catalunya grant number 2009SGR859. 
The structure of the paper is the following: In Section 2 first we give the basic notions and the known results about Darboux's theory of integrability for autonomous polynomial differential systems in $\mathbb{C}^{n}$. The new results are presented in Subsection 2.2. More concretely, Theorem 5 improves the known results of Darboux integrability for autonomous polynomial systems. The examples in Subsection 2.3 illustrate these improvements. In Section 3 first we present the basic notions about Darboux first integrals and Jacobi multipliers for a class of non-autonomous vector fields. The new results for non-autonomous vector fields are given in Subsection 3.2, in Theorem 8. At the end we illustrate the theory with an example.

\section{DARboux FIRST integrals FOR AUtONomous DifFERENTIAL SYSTEMS}

2.1. Basic notions and known results. First, we will fix the following notation. Let $\mathcal{F}$ be a differential field of characteristic zero, and let us denote by $\partial$ its derivation. The field of constants of $\mathcal{F}$ is denoted by $C(\mathcal{F})$. We consider $a_{1}, \cdots, a_{r} \in \mathcal{F}$. We denote by $\mathcal{W}\left(a_{1}, \ldots, a_{r}\right)$ the Wronskian matrix

$$
\mathcal{W}\left(a_{1}, \cdots, a_{r}\right)=\left(\begin{array}{ccc}
a_{1} & \cdots & a_{r} \\
\partial a_{1} & \cdots & \partial a_{r} \\
& \cdots & \\
\partial^{(r-1)} a_{1} & \cdots & \partial^{(r-1)} a_{r}
\end{array}\right) .
$$

The following differential algebraic lemma contains the core of Darboux's method for the search of first integrals and Jacobi multipliers.

Lemma 1. Let us consider elements $f_{1}, \cdots, f_{r}, \psi \in \mathcal{F}$ and denote by $a_{i}=\left(\partial f_{i}\right) f_{i}^{-1}=$ $\partial \log f_{i}$. The following conditions are equivalent:

(i) The rank of the Wronskian matrices $\mathcal{W}\left(a_{1}, \ldots, a_{r}, \psi\right)$ and $\mathcal{W}\left(a_{1}, \ldots, a_{r}\right)$ coincide.

(ii) $\psi$ is a non-trivial $C(\mathcal{F})$-linear combination of $a_{1}, \ldots, a_{r}$

$$
\psi=\lambda_{1} a_{1}+\cdots+\lambda_{r} a_{r} .
$$

(iii) There exist constants $\lambda_{1}, \cdots, \lambda_{r}$ in $C(\mathcal{F})$ such that for any differential extension $\mathcal{F} \subset \mathcal{F}\left(b_{1}, \cdots, b_{r}\right)$ by elements $b_{i}$ satisfying $\partial b_{i}=\lambda_{i} a_{i} b_{i}$ the element $M=b_{1} \cdots b_{r}$ verifies $\partial M=\psi \cdot M$.

Proof. The equivalence between (i) and (ii) is due to Lemma 1.12 in [29]. The equivalence between (ii) and (iii) is proved using the same argument as in the proof of the classical theorem of Darboux, see [11]. We note that a simple computation shows that $\partial \log M=\sum_{i=1}^{r} \lambda_{i} a_{i}$.

\section{Remark 2.}

(i) If $\psi=0$, then $M$ is a new constant.

(ii) If $\partial$ represents the Lie derivative with respect a vector field of divergence $\psi$ then $M$ is a Jacobi multiplier.

We will develop this remark throughout the forthcoming sections.

We consider the $n \times n$ complex polynomial differential systems of the form

$$
\dot{x_{j}}=\frac{d x_{j}}{d t}=P_{j}\left(x_{1}, \cdots, x_{n}\right)=\sum_{0 \leq i_{1}+\cdots+i_{n} \leq m}^{n} a_{j i_{1} \cdots i_{n}} x_{1}^{i_{1}} \cdots x_{n}^{i_{n}}, \quad j=1, \cdots, n
$$


with $a_{j i_{1} \cdots i_{n}} \in \mathbb{C}$. Note that system (3) has degree $m=\max \left\{\operatorname{deg} P_{1}, \cdots, \operatorname{deg} P_{n}\right\}$. The associated vector field of system (3) is

$$
X=\sum_{i=1}^{n} P_{i} \frac{\partial}{\partial x_{i}} .
$$

Let $f \in \mathbb{C}\left[x_{1}, \cdots, x_{n}\right]$ be irreducible. The hypersurface $f=0$ is an invariant hypersurface of the vector field (4) if it satisfies

$$
X(f)=\sum_{j=1}^{n} P_{j} \frac{\partial f}{\partial x_{j}}=K f,
$$

or, equivalently,

$$
\frac{X(f)}{f}=X(\log (f))=K,
$$

with $K \in \mathbb{C}_{m-1}\left[x_{1}, \cdots, x_{n}\right]$. We stress that if the polynomial system (3) has degree $m$ then we have that $\operatorname{deg} K \leq m-1$, independently of the degree of the hypersurface $f\left(x_{1}, \cdots, x_{n}\right)=0$. Note that if an orbit $x(t)=\left(x_{1}(t), \cdots, x_{n}(t)\right)$ of the vector field $X$ has a point on the hypersurface $f=0$ then the whole orbit is contained in $f=0$. This is the reason that we say that the algebraic hypersurface is invariant for the vector field $X$, i.e. it is invariant by its flow.

Assume that $h, g \in \mathbb{C}\left[x_{1}, \cdots, x_{n}\right]$ and are relatively prime polynomials in the variables $x_{1} \cdots x_{n}$. The function $F\left(x_{1}, \cdots, x_{n}\right)=\exp (g / h)$ is called an exponential factor (see also [5]) of the differential system (3) if there is $K \in \mathbb{C}_{m-1}\left[x_{1}, \cdots, x_{n}\right]$ that satisfies

$$
X(F)=\sum_{j=1}^{n} P_{j} \frac{\partial F}{\partial x_{j}}=K F
$$

or equivalently

$$
X\left(\frac{g}{h}\right)=K
$$

and we say that $K$ is the cofactor of the exponential factor $F$. If $F=\exp (g / h)$ is an exponential factor with $g, h$ coprimes it is easy to prove that $h=0$ is an invariant hypersurface of $X$. The existence of exponential factors with cofactor of degree $\leq m-1$ is related with the concept of multiplicity $[9,19,20,21]$.

Let $W$ be an open subset of $\mathbb{C}^{n}$. An analytic function $I: W \longrightarrow \mathbb{C}$ is a first integral of the vector field $X$ on $\mathrm{W}$ if $X(I)=0$. This implies that $I$ is constant along integral curves of $X$ contained in $W$.

An analytic function $M: W \longrightarrow \mathbb{C}$ is a Jacobi multiplier of the vector filed $X$ on $W$ if it satisfies $X(M)=-(\operatorname{div} X) M$ where $\operatorname{div} X=\sum_{i=1}^{n} \partial P_{i} / \partial x_{i}$ is the divergence of the vector field (4). Note that due its definition a Jacobi multiplier is also an invariant hypersurface (maybe not a polynomial) of system (3) with cofactor $-\operatorname{div} X$. Additionally, the existence of a Jacobi multiplier $M$ yields to the divergence free system $M X=\left(M P_{1}, \cdots, M P_{n}\right)$. 
Consider $f_{1}, \cdots, f_{p}$ irreducible and coprime polynomials in $\mathbb{C}[x]$ and some $F_{1}, \cdots, F_{q}$ exponential factors. Any function of the form

$$
I=f_{1}^{\lambda_{1}} \cdots f_{p}^{\lambda_{p}} F_{1}^{\mu_{1}} \cdots F_{q}^{\mu_{q}},
$$

will be called a Darboux function. Note that powers and products of exponential factors are also exponential factors. Therefore any Darboux function can be written into the form

$$
I=f_{1}^{\lambda_{1}} \cdots f_{p}^{\lambda_{p}} \exp \left(\frac{g}{h}\right) .
$$

A Darboux first integral (Darboux Jacobi multiplier) is a first integral (Jacobi multiplier) given by a Darboux function of the form (8).

The following theorem summarizes known results on the subject of Darboux first integrals dating back to Darboux [10], Jouanolou [13], and some more recent works like $[5,6,19,27]$ among others.

Theorem 3. Assume that the polynomial vector field $X$ in $\mathbb{C}^{n}$ given by (4) of degree $m$ admits

(i) $p$ irreducible and coprimes invariant algebraic hypersurfaces $f_{1}=0, \cdots, f_{p}=$ 0 with cofactors $K_{1}, \cdots, K_{p}$.

(ii) $q$ exponential factors $F_{p+1}, \cdots, F_{p+q}$ with cofactors $K_{p+1}, \cdots, K_{p+q}$ of degree $\leq m-1$.

Then the following statements hold.

(a) If $p+q=\left(\begin{array}{c}n+m-1 \\ n\end{array}\right)+1$, then the vector field $X$ has a Darboux first integral.

(b) If $p+q=\left(\begin{array}{c}n+m-1 \\ n\end{array}\right)+n$ then the vector field $X$ has a rational first integral.

(c) If $p+q=\left(\begin{array}{c}n+m-1 \\ n\end{array}\right)$, then the vector field $X$ has either a Darboux first integral or a Darboux Jacobi multiplier.

Statement (a) is due to Darboux [10, 11], and statement (b) of Theorem 3 was proved by Jouanolou [13]. For a short proof of statement (b) see [5, 6] for $\mathrm{n}=2$ and [19] for $n \geq 2$. A similar to statement (c) of Theorem 3 appears in [1] and its proof follows easily.

Remark 4. In Theorem 3 the number $p+q \geq\left(\begin{array}{c}n+m-1 \\ n\end{array}\right)$ is a sufficient but not necessary condition for the existence of a Darboux first integral or of Jacobi multiplier.

2.2. New results for autonomous polynomial differential systems. Let $X$ be a polynomial vector field in $\mathbb{C}^{n}$. We consider $K_{1}, \cdots, K_{r}$ cofactors of hypersurfaces or of exponential factors of $X$. We denote by $\mathcal{W}$ the Wronskian matrix of the cofactors defined as

$$
\mathcal{W}=\mathcal{W}\left(K_{1}, \cdots, K_{r}\right)=\left(\begin{array}{ccc}
K_{1} & \cdots & K_{r} \\
X\left(K_{1}\right) & \cdots & X\left(K_{r}\right) \\
& \cdots & \\
X^{(r-1)}\left(K_{1}\right) & \cdots & X^{(r-1)}\left(K_{r}\right)
\end{array}\right)
$$


where $X^{(l+1)}\left(K_{j}\right)=X\left(X^{(l)}\left(K_{j}\right)\right)$. Next we are going to use the Wronskian (9) in order to present a result related to the classical Darboux Theorem (3).

Theorem 5. Assume that the polynomial vector field $X$ in $\mathbb{C}^{n}$ given by (4) of degree $m$ admits

(i) $p$ irreducible and coprime invariant hypersurfaces, $f_{1}=0, \cdots, f_{p}=0$ with cofactors $K_{1}, \cdots, K_{p}$.

(ii) $q$ exponential factors $F_{p+1}, \cdots, F_{p+q}$ with cofactors $K_{p+1}, \cdots, K_{p+q}$.

We further assume that the 1 -forms $d \log f_{1}, \ldots, d \log f_{p}, d \log F_{p+1}, \cdots, d \log F_{p+q}$ are linearly independent over $\mathbb{C}$.

(a) $p+q>\operatorname{rank}\left(\mathcal{W}\left(K_{1}, \cdots, K_{p+q}\right)\right)$ if and only if the vector field $X$ admits a first integral which is either rational or Darboux.

(b) $p+q>\operatorname{rank}\left(\mathcal{W}\left(K_{1}, \cdots, K_{p+q}\right.\right.$, div $\left.\left.X\right)\right)+1$ if and only if the vector field $X$ admits either a rational first integral or a Darboux Jacobi multiplier.

(c) $p+q>\operatorname{rank}\left(\mathcal{W}\left(K_{1}, \cdots, K_{p+q}\right)\right)+n$ then the vector field $X$ has at least one rational first integral.

Proof. The proof follows directly from Lemma 1. Let us consider $\mathcal{F}=\mathbb{C}\left(x_{1}, \cdots, x_{n}\right)$ as a differential field endowed with the derivation $X$. The field of constants $C(\mathcal{F})$ is the field of rational first integrals of $X$. Let us prove statement (a). Condition (i) of Lemma 1 is satisfied with $\psi=0$ (see equation (2)). Assuming that there is not a non-constant rational first integral, then we have that there is a non-trivial linear combination over $\mathbb{C}, \lambda_{1} K_{1}+\cdots+\lambda_{p+q} K_{p+q}=0$ and therefore $I=f_{1}^{\lambda_{1}} \cdots F_{p+q}^{\lambda_{p+q}}$ is a new constant of the extension $\mathcal{F} \subset \mathcal{F}\left(f_{1}^{\lambda_{1}}, \cdots, F_{p+q}^{\lambda_{p+q}}\right)$, and therefore a first integral of $X$. This function $I$ is not a constant in $\mathbb{C}^{n}$ because of the linear $\mathbb{C}$-independence of $d \log f_{1}, \ldots, d \log f_{p}, d \log F_{p+1}, d \log F_{p+q}$.

Let us prove statement (b). Now, condition (i) of Lemma 1 is satisfied with $\psi=-\operatorname{div} X$. Following the same argument, by Lemma 1(iii) we have that $M=$ $f_{1}^{\lambda_{1}} \cdots F_{p+q}^{\lambda_{p+q}}$ verifies that $X M=(-\operatorname{div} X) M$ and so it is a Jacobi multiplier of $X$.

The proof of statement (c) will be given as a particular case of Theorem 8 .

\section{Remark 6.}

(i) We note that the hypothesis of independence guarantees that $d \log I$ is a non zero rational 1-form and therefore $I$ is a non-constant function.

(ii) In Theorem 5 there is no condition about the degree of the cofactors $K_{p+1}, \ldots$, $K_{p+q}$ of the exponential factors.

(iii) If $\operatorname{det}\left(\mathcal{W}\left(K_{1}, \ldots, K_{r}\right)\right) \neq 0$ means that there is not a Darboux first integral formed by the invariant hypersurfaces and exponential factors that corresponds to the cofactors $K_{1}, \cdots, K_{r}$. However, it maybe exist a Darboux first integral formed by a different collection of invariant hypersurfaces and exponential factors.

\subsection{Examples.}

Example 1. We consider the quadratic vector field,

$$
X=(\mu x+\beta x y) \frac{\partial}{\partial x}+(\lambda y+\alpha x y) \frac{\partial}{\partial y} .
$$


It is clear that $f_{1}=x, f_{2}=y$ are invariant algebraic curves with cofactors $K_{1}=$ $\mu+\beta y$ and $K_{2}=\lambda+\alpha x$ respectively. We also consider exponential factors $F_{3}=$ $e^{x}$ and $F_{4}=e^{y}$, with cofactors $K_{3}=x K_{1}$ and $K_{4}=y K_{2}$ of degree two. We have $\operatorname{rank} \mathcal{W}_{3}\left(K_{1}, K_{2}, K_{3}, K_{4}\right)=3$ and so according to Theorem $5(b)$ we obtain the Darboux first integral of the form $I=x^{\lambda} y^{-\mu} e^{\alpha x-\beta y}$. Note that Theorem 3 cannot been applied in this case.

Note that the exponential factor $e^{\alpha x-\beta y}$ is also invariant of $X$ with cofactor $K=$ $\alpha \mu x-\beta \lambda y$ of degree one and we have that $\operatorname{rank}\left(\mathcal{W}_{3}\left(K_{1}, K_{2}, K\right)\right)=2$ and also $\lambda K_{1}-\mu K_{2}+K=0$. Therefore we can apply both Theorems 3 and 5.

Example 2. We consider the polynomial differential system

$$
\begin{aligned}
& \dot{x}=x z\left(b x^{n_{1}} y^{n_{2}} z^{n_{3}}-n_{2}\right) \\
& \dot{y}=-y^{n_{2}+1} x^{n_{1}} z^{n_{3}}(\gamma c x+a z)+y\left(n_{3} \gamma x+n_{1} z\right) \\
& \dot{z}=\gamma x z\left(b x^{n_{1}} y^{n_{2}} z^{n_{3}}-n_{2}\right),
\end{aligned}
$$

with $n_{1}, n_{2}, n_{3}$ positive integers and $a, b, c \in \mathbb{C}$ of degree $n_{1}+n_{2}+n_{3}+2$. Easy computations shows that system (10) admits the invariant surfaces $f_{1}=x, f_{2}=y$, $f_{3}=z$ with cofactors $K_{1}=b x^{n_{1}} y^{n_{2}} z^{1+n_{3}}-n_{2} z, K_{2}=-y^{n^{2}} 2 x^{n_{1}} z^{n_{3}}(\gamma c x+a z)+n_{1} z+$ $n_{3} \gamma x$ and $K_{3}=\gamma x\left(b x^{n_{1}} y^{n_{2}} z^{n_{3}}-n_{2}\right)$ respectively. Additonally, admits the exponential factor $F=\exp \left(\frac{1}{x^{n_{1}} y^{n_{2}} z^{n_{3}}}\right)$ with cofactor $K=-n_{1} b z+n_{2} \gamma c x+n_{2} a z-\gamma n_{3} b x$. We have $\operatorname{rank}\left(\mathcal{W}_{3}\left(K_{1}, K_{2}, K_{3}\right)\right)=2$ and therefore we can apply Theorem 5 (a). We obtain the first integral

$$
H(x, y, z)=x^{a} y^{b} z^{c} \exp \left(\frac{1}{x^{n_{1}} y^{n_{2}} z^{n_{3}}}\right) .
$$

Note that for system (10) we have $n=2, m=n_{1}+n_{2}+n_{3}+2$ and so

$$
\left(\begin{array}{c}
n+m-1 \\
n
\end{array}\right)=\frac{\left(n_{1}+n_{2}+n_{3}+2\right)\left(n_{1}+n_{2}+n_{3}+3\right)}{2} .
$$

Therefore for the application of Theorem 3 we need an arbitrarily big number of invariant surfaces and exponential factors.

\section{DARboux FIRST INTEGRALS FOR NON-AUTONOMOUS DIFFERENTIAL SYSTEMS}

3.1. Basic notions. Let $U$ be a connected open subset of $\mathbb{C}$. We consider $\mathcal{C}^{\omega}(U, \mathbb{C})$ the ring of analytic functions and we denote by $M(U, \mathbb{C})=\left\{f / g: f, g \in \mathcal{C}^{\omega}(U, \mathbb{C})\right\}$ the field of meromorphic functions in $U$. We recall that the derivative of a meromorphic function is also a meromorphic function and so the field $M(U, \mathbb{C})$ endowed with the derivation operator $d / d t$ is also a differential field.

We also consider the ring of polynomials in the variables $x_{1}, \cdots, x_{n}$ with coefficients in $M(U, \mathbb{C})$ and we denote it by $M(U, \mathbb{C})[x]=M(U, \mathbb{C})\left[x_{1}, \cdots, x_{n}\right]$. In particular $M(U, \mathbb{C})[x]$ is a domain of unique factorization. In the following we denote by $M(U, \mathbb{C})(x)$ the ring of rational functions in the variables $x_{1} \cdots x_{n}$ and coefficients in $M(U, \mathbb{C})$. Here, we deal with the differential systems of the form

$$
\dot{x_{j}}=\frac{d x_{j}}{d t}=P_{j}(t, x)=\sum_{0 \leq i_{1}+\cdots+i_{n} \leq m}^{n} a_{j i_{1} \cdots i_{n}}(t) x_{1}^{i_{1}} \cdots x_{n}^{i_{n}}, \quad j=1, \cdots, n
$$

with $a_{j i_{1} \cdots i_{n}} \in M(U, \mathbb{C})$ and so $P_{j} \in M(U, \mathbb{C})[x]$ for $j=1, \cdots, n$. Note that system (11) is a polynomial differential system in the variables $x_{1}, \cdots x_{n}$ of degree 
$m=\max \left\{\operatorname{deg} P_{1}, \cdots, \operatorname{deg} P_{n}\right\}$. In what follows these systems will be called nonautonomous polynomial differential systems. Note that $t$ is the independent variable of system (3) called the time.

We associate to the differential system (11) the vector field in $U^{*} \times \mathbb{C}^{n}$,

$$
X=\frac{\partial}{\partial t}+\sum_{j=1}^{n} P_{j} \frac{\partial}{\partial x_{j}} .
$$

where $U^{*}$ is the open subset of $\mathbb{C}$ obtained by removing from $U$ the poles of the coefficients of the polynomials $P_{i}$. The vector field $X$ can be interpreted as a derivation of the ring $M(U, \mathbb{C})[x]$ and therefore of the quotient field $M(U, \mathbb{C})(x)$. Thus, we obtain a differential field extension $(M(U, \mathbb{C}), d / d t) \subset(M(U, \mathbb{C})(x), X)$.

Let $f_{1}, \cdots, f_{p} \in M(U, \mathbb{C})[x]$ be irreducible and coprime polynomials. We also consider $F=\exp (g / h)$ with $g, h \in M(U, \mathbb{C})[x]$ coprimes.

Any function of the form

$$
f_{1}^{\lambda_{1}} \cdots f_{p}^{\lambda_{p}} \exp \left(\frac{g}{h}\right)
$$

will be called a generalized Darboux function.

Let $W$ be an open subset of $U \times \mathbb{C}^{n}$. A non constant analytic function $I: W \longrightarrow \mathbb{C}$ is a first integral of the vector field $X$ on $\mathrm{W}$ if it is constant on all integral curves $x(t)=\left(t, x_{1}(t), \cdots, x_{n}(t)\right)$ of $X$ contained in W. Hence $I(x(t))=$ constant for all values of $\mathrm{t}$ for which the solution $x(t)$ is defined and contained in $W$. So, $X(I)=0$ on $W$.

A nonconstant analytic function $M: W \longrightarrow \mathbb{C}$ is a Jacobi multiplier of the vector filed $X$ on $W$ if it satisfies $X(M)=-(\operatorname{div} X) M$ where $\operatorname{div} X=\sum_{i=1}^{n} \partial P_{i} / \partial x_{i}$ is the divergence of the vector field (12). Note that due its definition a Jacobi multiplier is also an invariant hypersurface (maybe not a polynomial) of system (11) with cofactor $-\operatorname{div} X$. Additionally, the existence of a Jacobi multiplier $M$ yields to the divergence free system $M X=\left(M, M P_{1}, \cdots, M P_{n}\right)$.

For non-autonomous systems (11) a Darboux first integral is a first integral given by a generalized Darboux function (13) and similarly forDarboux Jacobi multiplier.

The theory of Darboux integrability for a 2-dimensional non-autonomous polynomial systems has been developed in [17].

3.2. New results for non-autonomous polynomial differential systems. In what follows we consider the differential system $X$ in $U^{*} \times \mathbb{C}^{n}$ given by expression (12). First we prove the following lemma.

Lemma 7. Let $W$ be an open subset of $U \times \mathbb{C}^{n}$. Let $J_{1}, \cdots, J_{n}$ be functionally independent analytic first integrals of $X$ on $W$.

(a) If $J_{n+1}$ is another first integral then there exists analytic functions $C_{1}, \cdots, C_{n}$ such that:

$$
d J_{n+1}=C_{1} d J_{1}+\cdots+C_{n} d J_{n} .
$$

(b) The functions $C_{1}, \ldots, C_{n}$ are first integrals of $n$. 
(c) Assume $J_{k}=\log \left(I_{k}\right)$ for $k=1, \ldots, n+1$ where $I_{1}, \ldots, I_{n+1}$ are Darboux first integrals in which the factors $f_{1}, \ldots, f_{p}, F_{p+1}, \ldots, F_{p+q}$ appear, being $d \log f_{1}, \ldots, d \log f_{p}, d \log F_{p+1}, \ldots, d \log F_{p+q} \mathbb{C}$-linearly independent. Assume that at least one factor appearing in $I_{n+1}$ does not appear in the $I_{1}, \ldots, I_{n}$. Then, the functions $C_{i}$ are in $M(U, \mathbb{C})(x)$ and at least one of them is not a constant.

Proof. (a) The vector field $X$ has no zeroes inside $W$. It follows that at each point of $W$ the 1 -forms $d J_{1}, \ldots, d J_{n}$ are a basis of the space of 1 -forms anihilated by $X$. The functions $C_{1}, \ldots, C_{n}$ appearing in (14) are simply the coordinates of $d J_{n+1}$ in the above basis.

(b) Since the functions $J_{k}$ are first integrals, the Lie derivatives $\operatorname{Lie}_{X}\left(d J_{k}\right)$ vanish. The Lie derivative safisfies the Leibniz formula, and therefore by derivation of (14) we have,

$$
0=\sum_{k=1}^{n} \operatorname{Lie}_{X}\left(C_{k} d J_{k}\right)=\sum_{k=1}^{n} X\left(C_{k}\right) d J_{k} .
$$

Taking into account that $J_{1}, \ldots, J_{n}$ are functionally independent it follows that $X\left(C_{k}\right)=0$ for $k=1, \ldots, n$.

(c) First, if the functions $I_{k}$ are of Darboux type, it follows that $d J_{k}=I_{k}^{-1} d I_{k}$ are 1-forms,

$$
d J_{k}=f_{k 0} d t+\sum_{j=1}^{n} f_{k j} d x_{j}
$$

were the coefficients $f_{k i}$ are in $M(U, \mathbb{C})(x)$. The functions $C_{k}$ are the coordinates of $d J_{k+1}$ in the basis $d J_{1}, \ldots, d J_{n}$. They are obtained by solving a linear systems of equations with coefficients in $M(U, \mathbb{C})(x)$, and therefore they are in $M(U, \mathbb{C})(x)$. Let us assume now that all the functions $C_{k}$ are constant. By hypothesis, the 1 -forms $d J_{1}, \ldots, d J_{n+1}$ are $\mathbb{C}$-linear combinations of $d \log f_{1}, \ldots$, $d \log f_{p}, d \log F_{p+1}, \ldots, d \log F_{p+q}$. Substituting them in equation (14) it leads us to a non-trivial vanishing $\mathbb{C}$-linear combination of $d \log f_{1}, \ldots, d \log f_{p}, d \log F_{p+1}, \ldots$, $d \log F_{p+q}$. By hypothesis those 1-forms are $\mathbb{C}$-linearly independent, and therefore at least on of the functions $C_{k}$ is not in $\mathbb{C}$.

The following theorem generalizes the Darboux theory of integrability for $n^{-}$ dimensional non-autonomous polynomial systems.

Theorem 8. We assume that the differential system $X$ in $U \times \mathbb{C}^{n}$ given by (11) of degree $m$ admits

(i) $p$ invariant hypersurfaces $f_{1}=0, \cdots, f_{p}=0$ with cofactors $K_{1}, \cdots, K_{p}$. Here $f_{1}, \cdots, f_{p} \in M(U, \mathbb{C})[x]$ are irreducible and coprime polynomials.

(ii) $q$ exponential factors $F_{p+1}, \cdots, F_{p+q}$ with cofactors $K_{p+1}, \cdots, K_{p+q}$. Here $F_{i}=\exp \left(g_{i} / h_{i}\right)$ with $g_{i}, h_{i} \in M(U, \mathbb{C})[x]$ are irreducible and coprimes.

We further assume that $d \log f_{1}, \ldots, d \log f_{p}, d \log F_{p+1}, \ldots, d \log F_{p+q}$ are $\mathbb{C}$-linearly independent as meromorphic 1-forms in $U \times \mathbb{C}^{n}$.

Then the following statements hold.

(a) $p+q>\operatorname{rank}\left(\mathcal{W}\left(K_{1}, \cdots, K_{r}\right)\right)$ if there is a first integral. This first integral is either rational or Darboux (of the form (13)). 
(b) $p+q>\operatorname{rank}\left(\mathcal{W}\left(K_{1}, \cdots, K_{r}\right.\right.$, div $\left.\left.X\right)\right)+1$ if and only if there is a rational first integral or a Darboux Jacobi Multiplier of $X$ of the form (13).

(c) If $p+q>\operatorname{rank}\left(\mathcal{W}\left(K_{1}, \cdots, K_{r}\right)\right)+n$ for $j=1, \cdots, n$ then the vector field $X$ has at least one rational first integral.

Proof. Let us consider $\mathcal{F}=M(U, \mathbb{C})(x)$ endowed with the derivation $X$. The proof of statements (a) and (b) here follows exactly the same argument than Theorem 5 statements (a) and (b).

Let us prove statement (c). Reasoning by reductio ad absurdum let us assume that $\operatorname{rank}(\mathcal{W})<p+q-n$ and there is no rational first integral. Therefore, all minors of $\mathcal{W}$ of order $p+q-n$ vanish. Let us assume that there is a minor of order $p+q-n-1$ that does not vanish otherwise we can forget some of our invariant surfaces or exponential factors. Let us reorder our factors such that $\operatorname{det}\left(\mathcal{W}\left(K_{1}, \cdots, K_{p+q-n-1}\right)\right) \neq 0$ but for each $j=p+q-n-1, \cdots, p+q$ we have $\operatorname{det}\left(\mathcal{W}\left(K_{1}, \cdots, K_{p+q-n-1}, K_{j}\right)\right)=0$. The $n+1$ Wronskian matrices considered here are under the hypothesis of statement (a) and therefore they lead to $n+1$ different Darboux first integrals $I_{1}, \cdots, I_{n+1}$. The application of Lemma 7 to that set of first integrals leads us to the existence of a non-constant rational first integral. This completes the proof.

Remark 9. Note that the condition about the existence of a Darboux first integrals and Jacobi multipliers improve the conditions of Theorem 2 in [17].

Example 3. The integrability of autonomous three-dimensional Lotka-Volterra systems has been studied by many authors, see for example [3]. Here we consider the non-autonomous Lotka-Volterra system:

$$
\begin{aligned}
& \dot{x}=x\left(\frac{t a_{1}(t)-1}{t}-\frac{\lambda_{2} b_{21}+b_{31}}{\lambda_{1}} t x+b_{12} y+b_{13} z\right) \\
& \dot{y}=y\left(a_{2}(t)+b_{21} t x-\frac{\lambda_{1} b_{12}+b_{32}}{\lambda_{2}} y+b_{23} z\right) \\
& \dot{z}=z\left(-\lambda_{1} a_{1}(t)-\lambda_{2} a_{2}(t)+t b_{31} x+b_{32} y-\left(\lambda_{1} b_{13}+\lambda_{2} b_{23}\right) z\right) .
\end{aligned}
$$

System (15) admits the invariant hypersurfaces $f_{1}=x, f_{2}=y$ and $f_{3}=z$ with cofactors

$$
\begin{aligned}
& K_{1}=-\frac{\left(\lambda_{2} b_{21}+b_{31}\right)}{\lambda_{1}} t x+b_{12} y+b_{13} z+\frac{t a_{1}(t)-1}{t} \\
& K_{2}=a_{2}(t)+b_{21} t x-\frac{\lambda_{1} b_{12}+b_{32}}{\lambda_{2}} y+b_{23} z \\
& K_{3}=-\lambda_{1} a_{1}(t)-\lambda_{2} a_{2}(t)+t b_{31} x+b_{32} y-\left(\lambda_{1} b_{13}+\lambda_{2} b_{23}\right) z .
\end{aligned}
$$

Note that $\operatorname{det}\left(\mathcal{W}\left(K_{1}, K_{2}, K_{3}\right)\right) \neq 0$.

System (15) admits the exponential factor $F=\exp \left(\lambda_{1} \ln (t)\right)$ with cofactor $K=$ $\lambda_{1} / t$ and we have that $\operatorname{det}\left(\mathcal{W}\left(K_{1}, K_{2}, K_{3}, K\right)\right)=0$. System (15) admits the Darboux first integral

$$
H=x^{\lambda_{1}} y^{\lambda_{2}} z t^{\lambda_{1}} .
$$

\section{REFERENCES}

[1] L.R. Berrone and H. Giacomini, Inverse Jacobi multipliers, Rend. Circ. Mat. Palermo II $52(2003) 77-130$

[2] T.C. Bountis, A. Ramani, B. Grammaticos And B. Dorizzi, On the complete and partial integrability of non-Hamiltonian systems, Phys. A 128 (1984), 268-288. 
[3] L. Cairó And J. Llibre, Darboux integrability for 3D Lotka-Volterra systems, J. Phys. A 33 (2000), no. 12, 2395-2406.

[4] F. Cantrijn and W. Sarlet, Generalizations of Noether's theorem in classical mechanics, SIAM Rev. 23 (1981), 467-494.

[5] C. Christopher And J. Llibre, Algebraic aspects of integrability for polynomial systems, Qualitative Theory of Dynamical Systems 1 (1999), 71-95.

[6] C. Christopher And J. Llibre, Integrability via invariant algebraic curves for planar polynomial differential systems, Annals of Differential Equations 16 (2000), 5-19.

[7] C. Christopher, J. Llibre, Ch. Pantazi, X. Zhang, Darboux integrability and invariant algebraic curves for planar polynomial systems. J. Phys. A 35 (2002) 2457-2476.

[8] C. Christopher, J. Llibre, Ch. Pantazi, S. Walcher, Inverse problems for invariant algebraic curves: Explicit computations, Proc. Roy.Soc. Edinburgh Sect. A 139 (2009) 287302.

[9] C. Christopher, J. Llibre, J. V. Pereira, Multiplicity of invariant algebraic curves in polynomial vector fields, Pacific J. Math. 229 (2007) no.1, 63-117.

[10] G. Darboux, Mémoire sur les équations différentielles algébriques du premier ordre et du premier degré (Mélanges), Bull. Sci. math. 2ème série 2 (1878), 60-96; 123-144; 151-200.

[11] G. Darboux, De l'emploi des solutions particulières algébriques dans l'intégration des systèmes d'équations différentielles algébriques, C. R. Math. Acad. Sci. Paris 86 (1878), 1012-1014.

[12] J. Giné And J. Llibre, A family of isochronous foci with Darboux first integral, Pacific J. Math. 218 (2005), 343-355.

[13] J.P. Jounnolou, Equations de Pfaff algébriques, in Lectures Notes in Mathematics 708, Springer-Verlag, New York/Berlin, 1979.

[14] P.D. LAX, Integrals of Nonlinear Equations of Evolution and Solitary Waves, Commun. Pure Appl. Math. 21 (1968), 467-490.

[15] J. LLIBRE, Integrability of polynomial differential systems, in Handbook of differential equations, Elsevier, Amsterdam, 2004, pp. 437-532.

[16] J. Llibre and Ch. Pantazi, Polynomial differential systems having a given Darbouxian first integral, Bull. Sci. Math 128, (2004), 775-788.

[17] J. Llibre And Ch. PANTAZI, Darboux theory of integrability for a class of nonautonomous vector fields, J. Math. Phys., 50, (2009) 102705.

[18] J. Llibre And X. Zhang, Darboux integrability for the Rössler system, Internat. J. Bifur. Chaos Appl. Sci. Engrg. 12 (2002), no. 2, 421-428.

[19] J. Llibre And X. Zhang, Rational first integrals in the Darboux theory of integrability in $\mathbb{C}^{n}$, Bull. Sci. Math. 134 (2010), no. 2, 189-195.

[20] J. Llibre And X. Zhang, Darboux theory of integrability in $\mathbb{C}^{n}$ taking into account the multiplicity, J.Diff.Equat. 246, (2009), 541-551.

[21] J. LlibRe AND X. Zhang, Darboux theory of integrability for polynomial vector fields in $\mathbb{R}^{n}$ taking into account the multiplicity at infinity, Bull. Sci. Math. 133 (2009), no. 7, 765-778.

[22] J. Llibre And X. Zhang, Invariant algebraic surfaces of the Lorenz system. J. Math. Phys. 43 (2002), 1622-1645.

[23] J. Llibre And G. Rodríguez, Configurations of limit cycles and planar polynomial vector fields, J. Diff. Eqns. 198 (2004), 374-380.

[24] Juan J. Morales-Ruiz, Differential Galois Theory and Non-Integrability of Hamiltonian Systems. Birkhäuser, Basel 1999.

[25] M.J. Prelle and M.F. Singer, Elementary first integrals of differential equations, Trans. Amer. Math.Soc. 279 (1983) 613-636.

[26] P.J. Olver, Applications of Lie groups to differential equations, Springer, New York, 1986.

[27] D. Schlomiuk, Algebraic particular integrals, integrability and the problem of the center, Trans. Amer. Math. Soc. 338 (1993), 799-841.

[28] M.F. Singer, Liouvillian first integrals of differential equations, Trans. Amer. Math. Soc. 333 (1992) 673-688.

[29] M. van der Put And M. F. Singer, Galois theory of linear differential equations, Grundlehren der Mathematischen Wissenschaften [Fundamental Principles of Mathematical Sciences], 328. Springer-Verlag, Berlin, 2003. 
${ }^{1}$ Instituto de Matemáticas y sus Aplicaciones - Universidad Sergio Arboleda, Calle 74 no. 14-14, Bogotá, Colombia.

E-mail address: david@ima.usergioarboleda.edu.co

2 Departament de Matemàtica Aplicada I, Universitat Politècnica de Catalunya, (EPSEB), Av. Doctor Marañón, 44-50, 08028 Barcelona, Spain

E-mail address: chara.pantazi@upc.edu 